\title{
On Finding Two-connected Subgraphs in Planar Graphs
}

\author{
J. Scott Provan \\ Department of Operations Research \\ University of North Carolina \\ Chapel Hill, NC 27599-3180 ${ }^{1}$
}

UNC/OR TR94-15

December 1996

\begin{abstract}
We consider a basic problem in the design of reliable networks, namely, that of finding a minimum-weight 2-connected subgraph spanning a given set $K$ of vertices in a planar graph. We show a relationship between this problem and that of finding shortest trails and cycles enclosing $K$, and derive a polynomial-time algorithm in the case when the vertices of $K$ all lie on the same face.
\end{abstract}

Key words: network survivability; Steiner tree; planar graph; two-connected

${ }^{1}$ Supported in part by NSF grant No. CCR-9200572 
Network designers are becoming more aware of the necessity for buiding survivability into the design of a transportation or communications network [9]. This involves constructing the network so as to withstand a certain degree of failure in its vertices or edges and still continue to provide the required degree of performance. We consider in this note a basic survivability criterion for a network: namely, that its terminal vertices can continue to communicate after the failure of any one edge (any one vertex) in the network. With this in mind we define the 2-Edge-Connected (2-Vertex-Connected) Steiner Tree Problem - which will be referred to as 2EST (2VST) - to have as input $(G, \boldsymbol{w}, K)$, where $G=(V, E)$ is an undirected graph, $\boldsymbol{w}=\left(w_{e}: e \in E\right)$ is a set of nonnegative edge weights, and $K$ is a set of terminal vertices in $G$. The objective is to find the minimum weight subgraph $B$ of $G$ which spans $K$ and is 2-edge-connected (2-vertex-connected). 2-connected Steiner tree problems have enjoyed considerable attention $[2,5,7,8,9,13]$. Both 2EST and 2VST are known to be NP-complete, even when $G$ is planar, $K=V$, and all weights are 1 , since they can be used to determine the existence of a Hamiltonian circuit in a planar graph [4].

The standard Steiner tree problem has the same input as 2EST and 2VST, but the solution subgraph is required to be merely a spanning (1-connected) subgraph for $K$ (the vertex and edge versions in this case are the same). This problem has been studied quite extensively; see [6] for a full account. It is also NP-complete when $G$ is planar, but does have a polynomial-time solution when in $G$ is $K$-planar - that is $G$ has some planar embedding with $K$ lying on the same face $[3,10]$.

For the remainder of the discussion we consider instances where $G$ is plane, that is, planar with a specified embedding in the plane. An enclosing walk for $K$ in $G$ is a closed path - with possibly repeated edges and vertices - whose enclosed region contains $K$. An enclosing trail (cycle) is an enclosing walk with no repeated edges (vertices). Shortest enclosing walks and cycles have been studied in [11], and shortest 
enclosing trails and cycles have been studied in [1]. In the latter paper a polynomialtime algorithm is found for the shortest enclosing trail and shortest enclosing cycle problems. In this paper, we show the relationship between 2EST (2VST) and finding shortest enclosing trails (cycles) for $K$ in $G$. Monma, Munson, and Pulleyblank in [8] note that in the case where $G$ is $K$-planar the solution to 2VST is a cycle passing through all vertices of $K$, and they wonder whether this fact can be used to find an efficient algorithm for 2VST in this case. We give an affirmative answer to their question. The main result of this note is the following:

Lemma Let $\Gamma$ be a shortest enclosing trail (cycle) for $K$ in plane graph $G$. If $\Gamma$ passes through all vertices of $K$, then it is in fact an optimal solution for DEST (2VST). In particular, if $K$ lies entirely on the exterior face of $G$ then the shortest enclosing trail (cycle) for $K$ in $G$ is the optimal solution to 2EST (2VST).

Proof For the first part of the theorem, let $B$ be an optimal solution to 2EST (2VST) for instance $(G, \boldsymbol{w}, K)$. Then the boundary of $B$ can be traversed clockwise via a noncrossing walk $W$, by starting at any boundary edge and iteratively choosing the next adjacent edge so that only the exterior of $B$ lies immediately to the left of $W$. By the construction of $W$ it follows that if $W$ has a repeated edge (vertex) $x$, then removal of $x$ will disconnect $B$ into vertex-nonempty components, contradicting the connectivity of $B$. Thus $W$ is a trail (cycle), and further, the weight of $B$ is at least as large as the weight of $W$. But since $\Gamma$ passes through all of the vertices of $K$ and has no repeated edges (vertices), then $\Gamma$ is feasible to 2EST (2VST), and since $\Gamma$ is the shortest enclosing trail (cycle), then its weight is no greater than that of $B$. Thus $\Gamma$ is in fact optimal to $2 \mathrm{EST}$ (2VST).

For the second part of the theorem, note that if $K$ lies on the exterior boundary of $G$ then any shortest enclosing trail (cycle) $\Gamma$ for $K$ must pass through all of the vertices of $K$, and so from the above discussion $\Gamma$ will be the optimal solution to 2EST 
$(2 \mathrm{VST})$.

Corollary 2EST and 2VST can be solved in $O\left(|V|^{3} \log |V|\right)$ time on instances where $G$ is $K$-planar.

Proof Let $F$ be the face containing all of the vertices of $K$. Reembed $G$ so that $F$ is the exterior face. Now use the $O\left(|V|^{3} \log |V|\right)$ algorithm in [1] to find the shortest enclosing trail (cycle) for $K$ with respect to this embedding. By the lemma this will be the optimal solution to 2EST (2VST).

We note that even if $G$ is not $K$-planar, the lemma in this paper gives an efficient way of constructing a possible solution to 2EST (2VST). Simply find a shortest enclosing trail (cycle) for $K$ in $G$, and check whether it in fact passes through all of the vertices of $K$. If so, then it is a solution to 2EST (2VST).

\section{Additional Remarks}

It would be useful to include negative edge weights when considering instances of 2EST or 2VST, since it may be profitable to include connections between other vertices when constructing the network. The importance of the nonnegativity assumption on efficient solutions to 2EST or 2VST depends upon how one wants these additional connections to be made. As it stands, the solution requires all pairs of spanned vertices to be 2-connected, not just those in $K$. If edge weights are nonnegative then the optimal solution can also be assumed to be minimal, so that any solution that 2 -connects $K$ will in fact 2-connect all of its spanned vertices. The addition of negative edge weights to either problem, however, renders the problem NP-complete even on $K$-planar graphs, again being reducible from the planar Hamiltonian circuit problem (briefly, by replacing each vertex by a cycle of negatively weighted edges, and setting $K=\emptyset)$. 
A variant of the problem would require that only the pairs of vertices of $K$ be 2-connected, and that any additional edges are of value solely with respect to the pair of vertices they connect. In particular, as long as $K$ is 2-connected any additional negatively-weighted edges can be added without violating the feasibility of the solution. This version of the problem can be reduced to the 2EST or 2VST problem with nonnegative weights: simply replace all of the negative weights by zeroes, find the optimal solution to 2EST or 2VST, and then add back any negatively weighted edges not already in the solution. The resulting set of edges is then optimal to the modified problem. The results in this paper again apply to the instance with the modified weights, and in particular this problem can also be solved in $O\left(|V|^{3} \log |V|\right)$ time on $K$-planar graphs.

One final potential application we need to address in this context is to the problem of finding Steiner hulls for 2EST and 2VST problems. A Steiner hull for an instance $(G, \boldsymbol{w}, K)$ of 2EST or 2VST is a subgraph $H$ of $G$ that is known to contain a solution to 2EST or 2VST; that is, any solution for instance $(H, \boldsymbol{w}, K)$ will also be a solution for instance $(G, \boldsymbol{w}, K)$. The motivation here is that using $H$ instead of $G$ considerably reduces the size of the graph in which the Steiner tree is found, perhaps even to the point where $H$ is $K$-planar. It turns out that the shortest enclosing walk for $K$ encloses a Steiner hull for the standard Steiner tree problem [10], and also for certain higher edge connectivity problems - including 2EST - in the case where an edge is allowed to appear multiple times (with corresponding multiple cost) in the optimal solution [12]. A analogous compelling supposition in view of the results of these papers and the treatment here would be that a shortest enclosing trail (cycle) $\Gamma$ for $K$ will enclose a Steiner hull for 2EST (2VST). This, unfortunately, is not the case, as is demonstrated in the instance given in the figure below. The terminal set is $K=\{f, g\}$. The unique shortest enclosing walk for $K$ - which also happens to be a trail and cycle - is 
$\Gamma=b-d-e-c-h-b$. The unique optimal solution for both 2EST and 2VST for this instance, however, is the cycle $b-a-c-g-e-d-f-b$, which is not enclosed by $\Gamma$. It does not appear, moreover, that the right embedding for $G$ will alleviate this problem in general. We leave to future research methods for constructing "good" Steiner hulls for 2EST and 2VST along these lines.

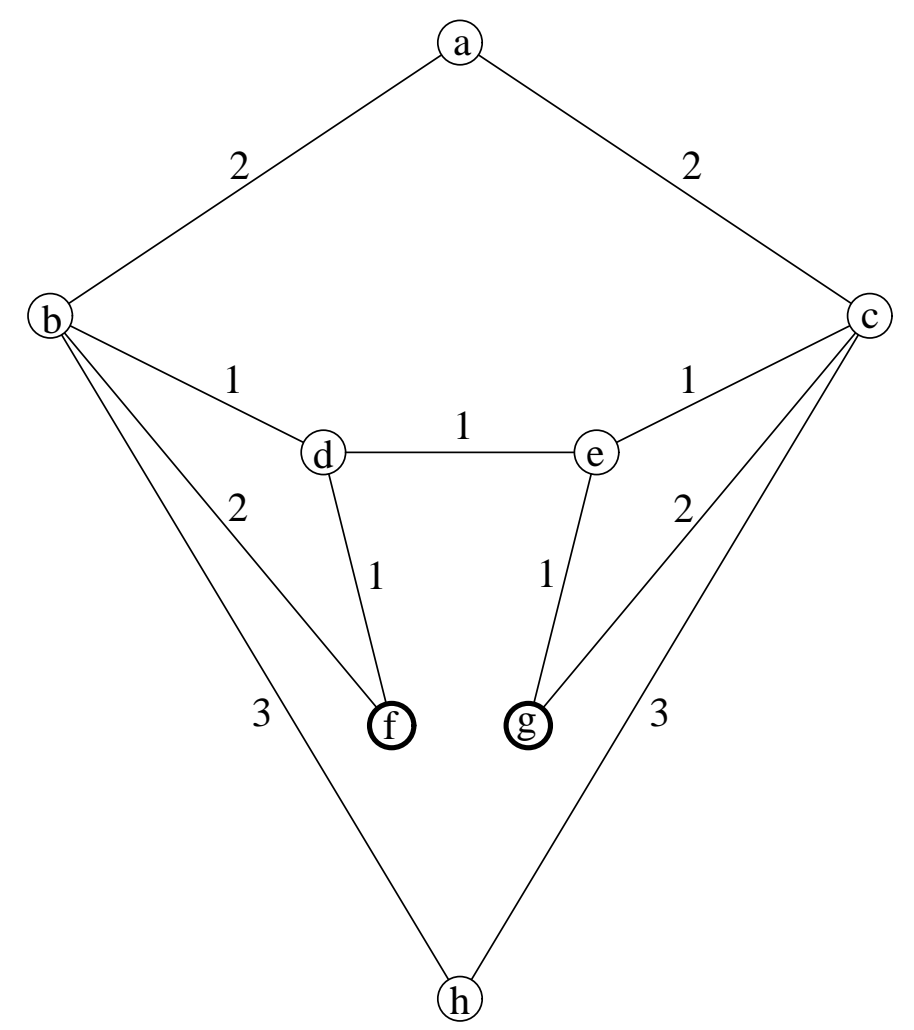

Figure: Counterexample

\section{References}

[1] C. Alexopoulos, J.S. Provan, H.D. Ratliff, and B.R. Stutzman, 1995. Minimal connected enclosures on an embedded planar graph, Technical Report UNC/OR/TR95-08, Department of Operations Research, University of North 
Carolina, Chapel Hill. (Postscript manuscript available by anonymous ftp under "Technical Reports" at WWW site http://www.or.unc.edu.)

[2] M. Baïou and A.R. Mahjoub, 1993. The D-edge connected Steiner subgraph polytope of a series-parallel graph, Technical Report, Département d'Informatique, Université de Bretagne Occidentale, Brest, France.

[3] R.E. Erickson, C.L. Monma, and A. F. Veinott, 1987. Send and split method for minimum-concave-cost network flows, Math. Oper. Res., 12, pp. 634-664.

[4] M.R. Garey, D.S. Johnson and R.E. Tarjan, 1976. The planar Hamilonian circuit problem is NP-complete, SIAM J. Computing 5. pp. 704-714.

[5] M. Grötschel and C.L. Monma, 1990. Integer polyhedra arising from certain network design problems with connectivity constraints, SIAM J. Discrete Math. 3, pp. 502-523.

[6] F. K. Hwang, D.S. Richards, and P. Winter, The Steiner Tree Problem, North-Holland, New York, NY, 1992.

[7] S. Khuller and U. Vishkin, 1994, Biconnectivity approximations and graph carvings, Jour. ACM 41, pp. 214-235.

[8] C.L. Monma, B.S. Munson, and W.R. Pulleyblank, 1990. Minimum-weight two-connected spanning networks, Math. Programming 46, pp. 153-171.

[9] C.L. Monma and D.F. Shallcross, 1989. Methods for designing communications networks with certain two-connected survivability constraints, Operations Research 37 , pp. 531-541.

[10] J. S. Provan, 1988. Convexity and the Steiner tree problem, Networks, 18, pp. $55-72$. 
[11] J.S. Provan, 1989. Shortest enclosing walks and cycles in embedded graphs, Information Processing Letters 30. pp. 119-125.

[12] K.T. Talluri, 1990. Convexity and the generalized Steiner problem, Technical Report, Operations Research Center. Massachsetts Institute of Technology, Cambridge, MA.

[13] P. Winter, 1986. Generalized Steiner problem in series-parallel networks, J. Algorithms 7, pp. 549-566. 